\title{
PERFORMANCE ANALYSIS OF SELECTED MEDICAL IMAGE SEGMENTATION ALGORITHMS.
}

\author{
Arulogun O.T., Falohun A.S., Ismaila W.O. \& Awokola J.A. \\ Department of Computer Science \& Engineering, Ladoke Akintola University of Technology, \\ Ogbomoso, Nigeria \\ Corresponding Authors E-mail: otarulogun@lautech.edu.ng, asfalohun@lautech.edu.ng, \\ woismaila@lautech.edu.ng
}

\begin{abstract}
The ability to understand images is one of the key areas of research where the target is to understand the components of an image and interpret its meaning. The components of images are not easily understood and interpreted for analysis, therefore image segmentation technique is very necessary and should be taken as significant step during image processing and analysis. Repeatable experiments are required for this research field to progress as image segmentation needed for data processing cannot be adequately achieved in a particular technique. Hence performance analysis of various, commonly used techniques are often needed. However, in this work, three classes of image segmentation algorithms: Edge Detection, Clustering and Thresholding method were simulated in MatLab on infected bladder. The experiments were performed three times to confirm the accuracy of the results evaluated with Dice, Jaccard and cosine co-efficient of fitness values.
\end{abstract}

\section{Keywords}

Segmentation, Bladder, Clustering, Detection, Thresholding.

\section{Academic Discipline and Sub-Discipline}

Computer Science, Computer Engineering, Information Systems.

\section{Subject Classification}

Pattern Recognition, Artificial Intelligence, Machine Learning.

\section{Type (Method / Approach)}

Experimentation, Computer Simulation.

\section{INTRODUCTION}

The use of images and image interpretation have formed a very wide field on its own. In recent time it has received much interest of researchers. Image interpretation have been used all over the world in so many fields by programmers, engineers, analysts, graphic designers, medical personnel, security experts and a whole lot of people through the interpretation of image contents which is one of the objectives in computer vision specifically in image processing. Segmentation helps in the separation of an image into its component regions or objects. Image segmentation needs to isolate the object from the background to read image properly and identify its content carefully. Image segmentation plays a significant role in computer vision today and all other related applications such as analysis of remote sensing data, analysis of medical data, and astronomical data as reported by [1] .It aims at extracting meaningful objects lying in the image, separating the image into various parts using different characteristics. Generally, there is no single method or approach for image segmentation but different techniques have been used over the years based on respective strengths and unique capabilities. Examples include Edge Detection techniques [1], clustering technique [2] cited in [3] and Thresholding principles [4].

\section{LITERATURE REVIEW}

\subsection{A Brief History of Image Segmentation}

Image segmentation is an important technique well known by its utility and complexity. To extract the useful information from images or groups of images, an inevitable step is to separate the objects from the background. [5] narrated the history of segmentation of digital images using computers to be traced back to 40 years ago and since then the field of image segmentation has evolved very quickly and has undergone great change. In 1965, an operator for detecting the edges between different parts of an image, Roberts operator (also called Roberts Edge Detector), was introduced and used for partition of image components. Over the last 40 years, the research and development of segmentation algorithms are going on and making very rapid progress.

\subsection{Approaches to Image Segmentation}

Due to the advent of various techniques and approaches to image segmentation, it has been an attractive topic amongst enthusiasts but equally challenging as an image represents various shapes, colors, intensities and this information should be preserved in processing. Though features can vary from images, there are few common features such as edge or boundary between backgrounds, segmentation is one of the useful methods for processing such [6]. There are various approaches to segmentation which include thresholding, edge based, region based segmentation and hybrid techniques. 


\subsubsection{Thresholding}

In thresholding-based segmentation, the image histogram can be partitioned into two classes using a single value (called bi-level thresholding) or multiple classes using multiple values (called multi-level thresholding) based on the characteristics of the histogram. In the bi-level thresholding, pixels with intensity values less than the threshold are set as background while others are set as object. [4] employed five thresholding techniques on three satellite images in a quest to find the best technique in Threshold segmentation. The Histogram Dependent Technique and the Edge Maximization Technique were reported as best.

\subsubsection{Edge Based Detection}

Edge detection techniques transform images to edge images benefiting from the changes of grey tones in the images. Edges are the sign of lack of continuity in image intensity, and ending. As a result of this transformation, edge image is obtained without encountering any changes in physical qualities of the main image. [7]. The edge representation of an image significantly reduces the quantity of data to be processed, yet it retains essential information regarding the shapes of objects in the scene. On the other hand, there is not yet any common performance directory to judge the performance of the edge detection techniques. The performance of edge detection techniques are always judged personally and separately dependent to its application. Of the various types of techniques used in Edge Based Detection are:

\subsubsection{Roberts Edge Algorithm}

It performs a simple, quick to compute, 2-D spatial gradient measurement on an image. This method emphasizes regions of high spatial frequency which often correspond to edges. [8]

\subsubsection{The Sobel Edge Algorithm}

The Sobel method of edge detection for image segmentation finds edges using the Sobel approximation to the derivative. It precedes the edges at those points where the gradient is highest. The Sobel technique performs a 2-D spatial gradient quantity on an image and so highlights regions of high spatial frequency that correspond to edges. [8]

\subsubsection{The Prewitt Edge Algorithm}

To estimate the magnitude and orientation of an edge, Prewitt is a correct way. Even though different gradient edge detection wants a quiet time consuming calculation to estimate the direction from the magnitudes in the $x$ and $y$-directions, the compass edge detection obtains the direction directly from the kernel with the highest response. [9]

\subsubsection{Fuzzy Algorithm}

The algorithm is based on the subjection of a set of four pixels, part of a $2 \times 2$ window of an image to a set of fuzzy conditions which help to highlight all the edges that are associated with an image. The fuzzy conditions help to test the relative values of pixels which can be present in case of presence on an edge. So the relative pixel values are instrumental in extracting all the edges associated to an image. The image is said to have an edge if the intensity variation in between the adjacent pixels is large. [10]. In 2009, Barkhoda proposed a fuzzy which experimental results show the higher quality and superiority of the extracted edges compared to the other methods in the literature such as Sobel, Robert and Prewitt. In the proposed algorithm, to achieve good result, some parameters and thresholds were experimentally set.

\subsubsection{Clustering Based Detection}

Clustering is an interesting approach for finding similarities in data and putting similar data into groups. Clustering algorithms are used extensively not only to organize and categorize data, but are also useful for data compression. The applicability of clustering methodology to the image segmentation problem was recognized over three decades ago, and the paradigms underlying the initial pioneering efforts are still in use today [11]. [12] analyzed the performance of cluster based algorithms for image segmentation with K-Means, improved K-Means, Fuzzy C-Means, improved Fuzzy C-Means and a proposed method and concluded that the proposed algorithm, the improved Fuzzy C-Means outperformed the others in terms of accuracy and convergence rate.

2.2.3.1 K-Means Algorithm (KM): The popular k-Means algorithm is an error minimization algorithm where the function to minimize is the sum of squared error. Two factors have made the k-Means popular: it has linear time complexity and its easy implementation. K-means is one of the simplest unsupervised learning algorithms that solve the well-known clustering problem. The procedure follows a simple and easy way to classify a given data set through a certain number of clusters (assume k clusters) fixed a priori. This algorithm aims at minimizing an objective function, vis-à-vis, a squared error function. [12]

2.2.3.2 Fuzzy C-Means Algorithm (FCM): One restriction of the k-Means algorithm is that it associates each pattern of the image into one, and only one, cluster. With the use of fuzzy theory, each pattern can be associated with every cluster using a membership function. Fuzzy c-means (FCM) is a method of clustering which allows one piece of data to belong to two or more clusters. That is, it allows the pixels belong to multiple classes with varying degrees of membership. [7].

\subsubsection{Efficient Graph-based Segmentation}

$7141 \mid \mathrm{P}$ a g e

July , 2016
Councilfor Innovative Research

w w w. cirworld. co m 
Efficient graph-based image segmentation is another method of performing clustering in feature space. This method works directly on the data points in feature space, without first performing a filtering step, and uses a variation on single linkage clustering. The key to the success of this method is adaptive thresholding.

\subsection{Bladder}

The bladder, also known as the urinary bladder is an expandable muscular sac that stores urine before it is excreted out of the body through the urethra. It is located in the lower abdominal area near the pelvic bones. Healthy bladders hold urine until people are able to relieve themselves but problem can arise for varying reasons.

\subsubsection{Bladder Infection}

A bladder infection also called cystitis is a bacterial infection within the bladder. It is also called urinary tract infection (UTI) or cystitis. This refers to a bacterial infection anywhere in the urinary tract which can be diagnosed by medical doctors through urine test, Kidney and Bladder Ultrasound, Computerized tomography (CT scan)) and examination of the bladder wall with a scope called cystoscope.

However, with pattern recognition, an attempt is made in this research to segment infectious bladder using some of the afore-mentioned categories of image segmentation schemes and compare the performance.

\section{METHODOLOGY}

\subsection{Image Acquisition}

The images to be used were acquired online at the link provided for figure 1 (bladder images). These images were acquired from composite database of websites and other image storage and linkage sites. The medical images were gotten through search engines and a search link. Although those images were not readily available but 20 images were gotten for the experiment. The selected images are of same size, in 2D without which incongruent results will be obtained.
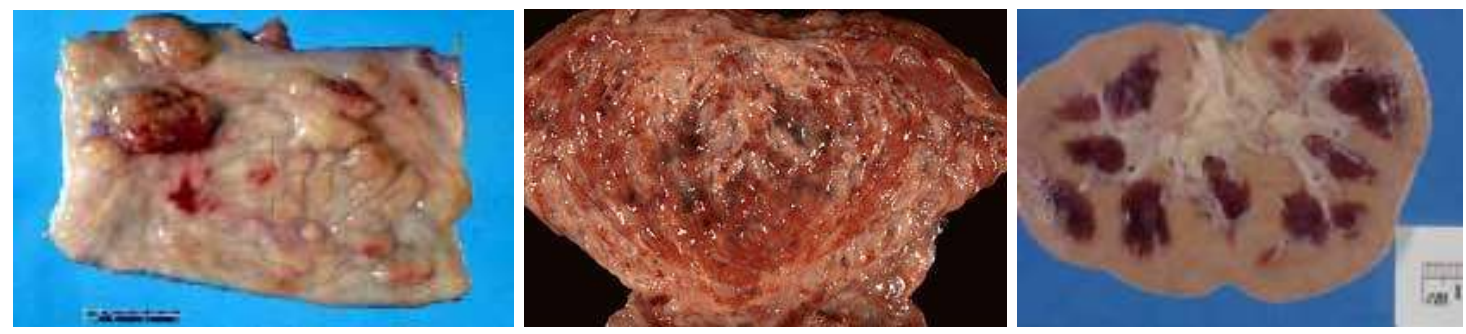

Figure 1: Bladder Images

Source: (www.google.com.ng/search?q=pictures+of+infected+bladder\&client=ms-opera-iniandroid\&channel=new\&tbm=isch\&prmd=ivns\&ei=n_8FV8KXC4vtatzBkNgD\&start=140\&sa=N)

\subsection{Image Pre-Processing}

The images were preprocessed in filtering, selecting, gray-scale conversion, resizing before segmentation.

\subsection{Image Database Creation}

After the images were obtained, a database was created for it in MatLab accordingly.
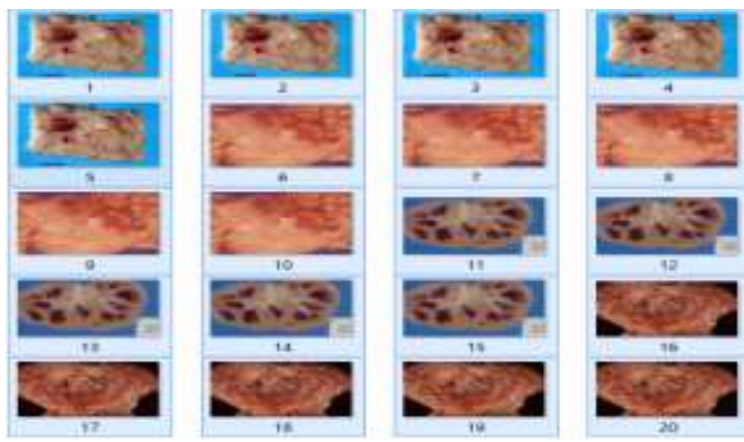

Figure 2: Processed bladder image database.

\subsection{Algorithm for Bladder Segmentation}

The proposed methods used are Edge Detection method, Cluster segmentation methods, and thresholding method of which three-types of algorithm are used under Edge Detection and two under Cluster segmentation. 


\subsubsection{Edge Detection Segmentation Method (1): Fuzzy Algorithm}

Step 1: Start

Step 2: Read image

Step 3: Convert image to gray scale and double-precision data

Step 4: Obtain image gradient

Step 5: Create fuzzy inference system (FIS) for edge detection

Step 6: Specify FIS rules

Step 7: Evaluate FIS

Step 8: Display results

Step 9: End.

\subsubsection{Edge Detection Segmentation Method (2): Prewitt Algorithm}

Step 1: Start

Step 2: Read Image

Step 3: Convert to Gray scale

Step 4: Apply Prewitt Edge Detection Function

Step 5: Display Result

Step 6: End.

\subsubsection{Edge Detection Segmentation Method (3): Sobel Algorithm}

Step 1: start

Step 2: Read Image

Step 3: Obtain the gradient of the Image

Step 4: Find the Magnitude

Step 5: Threshold the gradient Image

Step 6: Display Result

Step 7: End.

\subsubsection{Cluster Segmentation Method (1): K-means Algorithm}

Step 1: Start

Step 2: Read image

Step 3: Convert image from RGB color space to $I^{*} a^{*} b^{*}$ color space

Step 4: Cluster Image using k-means clustering

Step 5: Label every pixel in the image using the results from k-means

Step 6: Separate Object by Color.

Step 7: Segment the infected area into a separate image

Step 8: Display result

Step 9: End.

\subsubsection{Cluster Segmentation Method (2): Fuzzy C-mean Algorithm}

Step 1: Start

Step 2: Read Image

Step 3: Threshold Image and convert to $B / w$

Step 4: Performing Fuzzy C-Means Clustering

Step 5: Display Result 
Step 6: End.

\subsubsection{Thresholding Method}

Step 1: Start

Step 2: Read Image

Step 3: Convert Image to Grayscale

Step 4: Graythresh Image to get thresholding value

Step 5: Threshold Image

Step 6: Display Result

Step 7: End.

\subsection{Simulation}

The implementation of the algorithms was done using MatLab. R2015a, and computed on a 1.7-GHz computer system with $4 \mathrm{~GB}$ of memory.

\subsection{Evaluation Metrics.}

Dice coefficient:

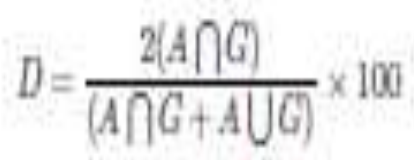

(1)

Jaccard coefficient:

Jaccard $=\frac{|X \cap Y|}{|X|+|Y|-|X \cap Y|}$

Cosine coefficient:

Cosine Coefficient $=\frac{|X \cap Y|}{|X|^{1 / 2} \cdot|Y|^{1 / 2}}$

\section{RESULT AND DISCUSSION}

Here is a presentation of the research findings and explanation.

\subsection{Results}

The result of each of the six algorithms used in the experiment with three different set of bladder images is shown in the Figures 3, 4 and 5.

The graphical comparison of the six algorithms was plotted and shown to determine the best fitness values. This was done for each of the images used and the mean results is shown in Table 1. Also, the time taken for each algorithm to complete the segmentation of the original image in seconds was also noted and reported appropriately in Table 2. 




Figure 3a. Bladder 1 Segmentation

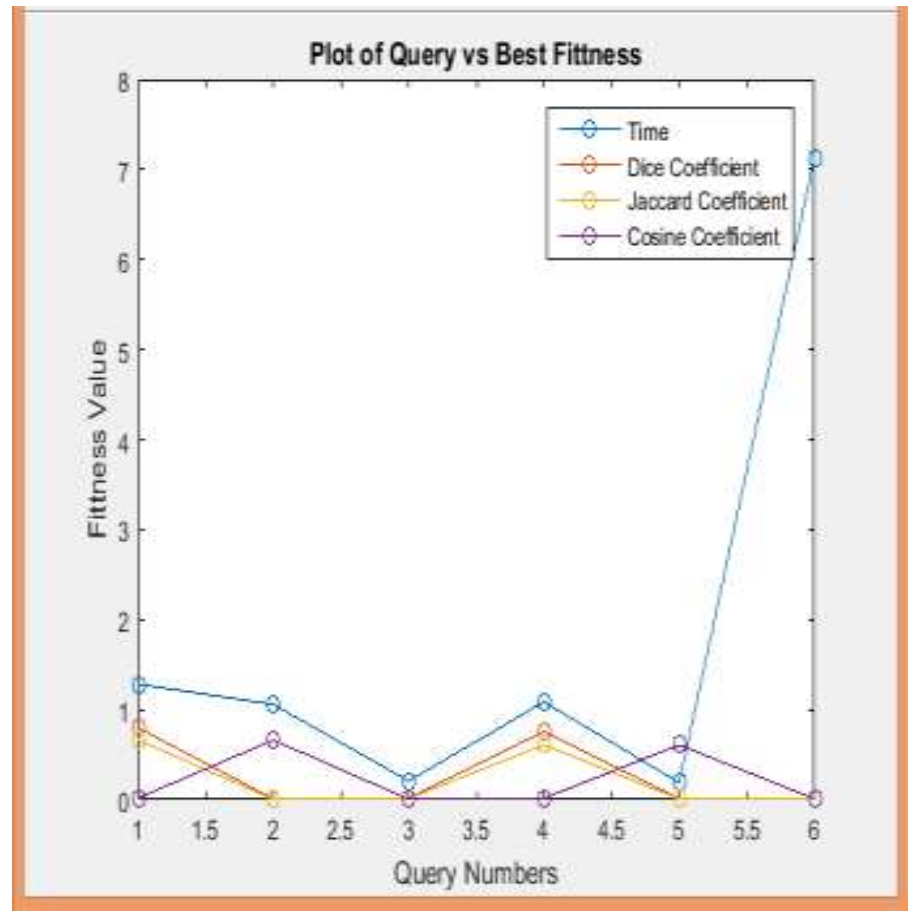

Figure 3b. Comparative segmentation results of the six algorithms on bladder Image 1. 


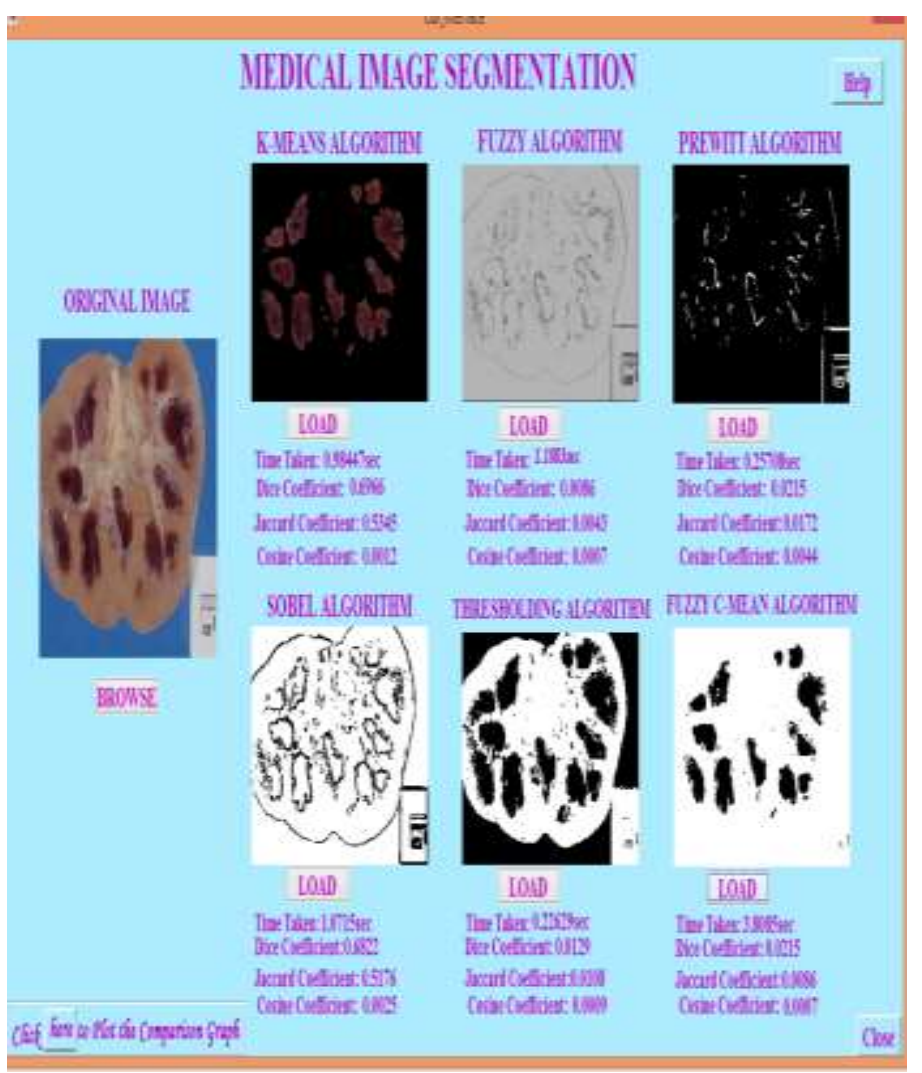

Figure 4a. Bladder 2 Segmentation

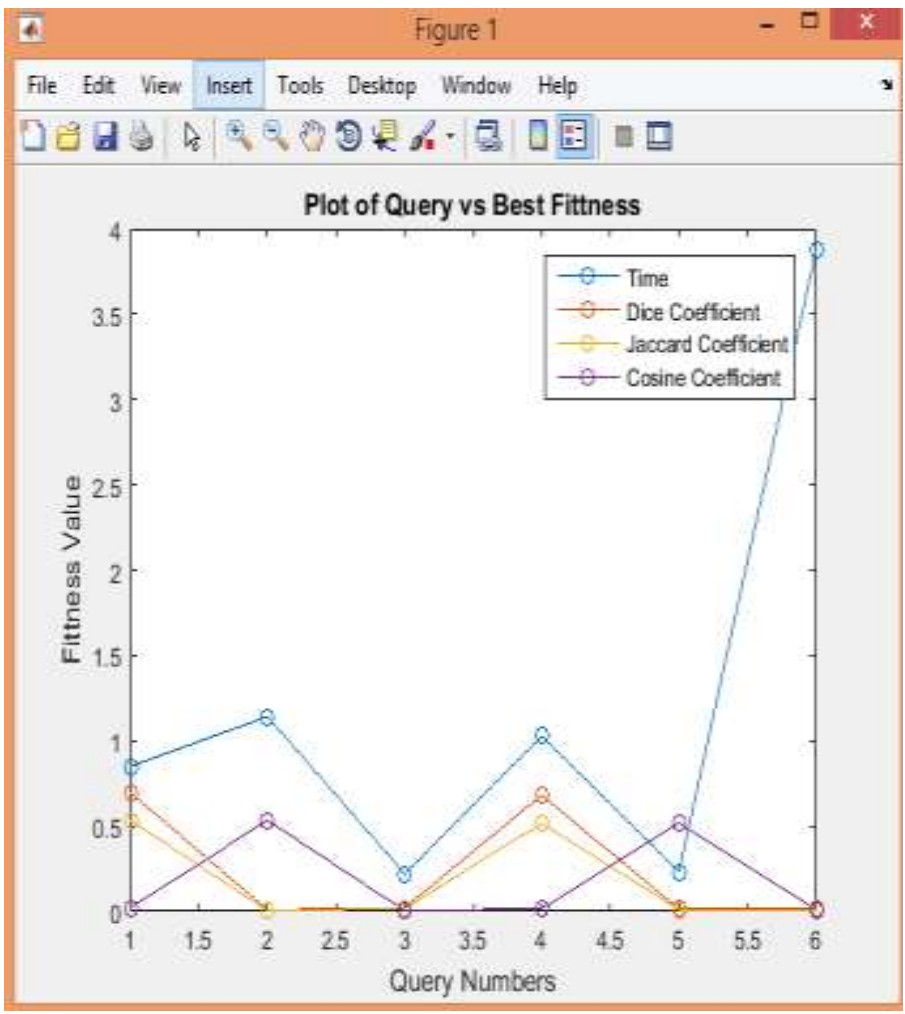

Figure 4b. Comparative segmentation results of the six algorithms on bladder Image 2 . 


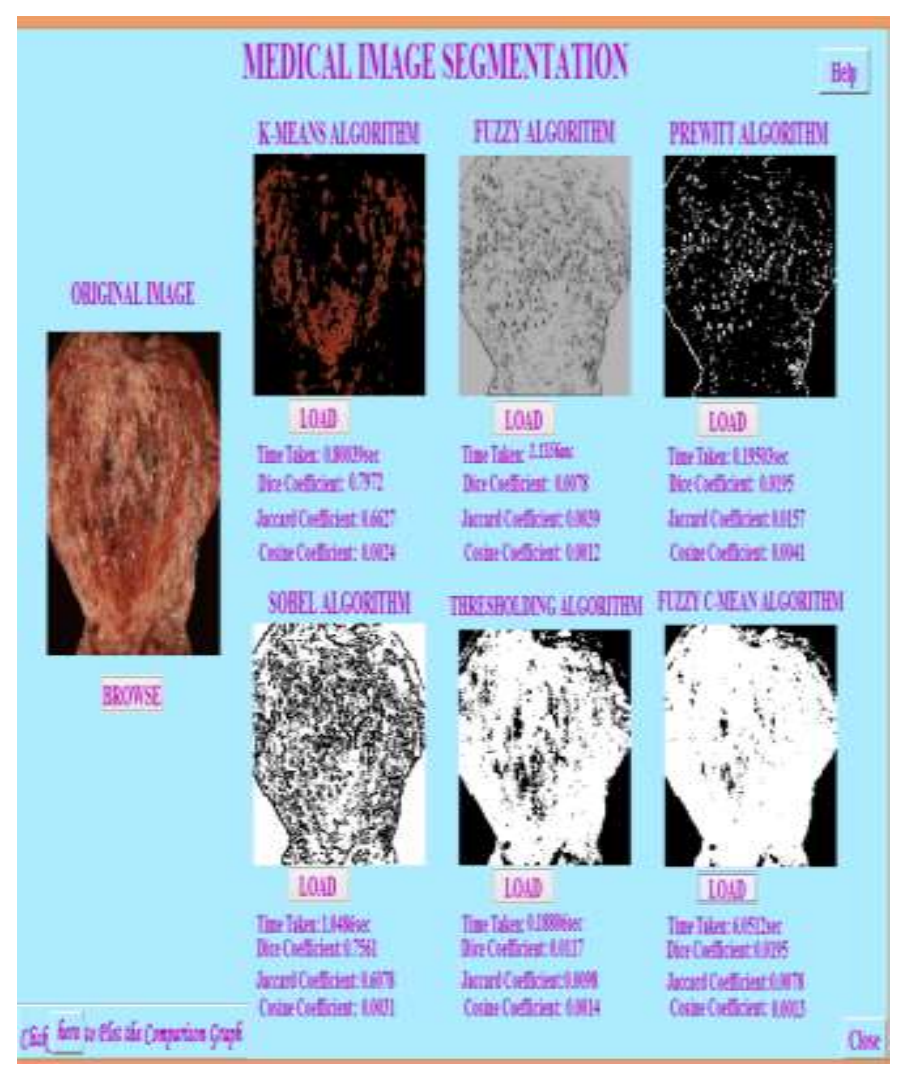

Figure 5a. Bladder 3 Segmentation

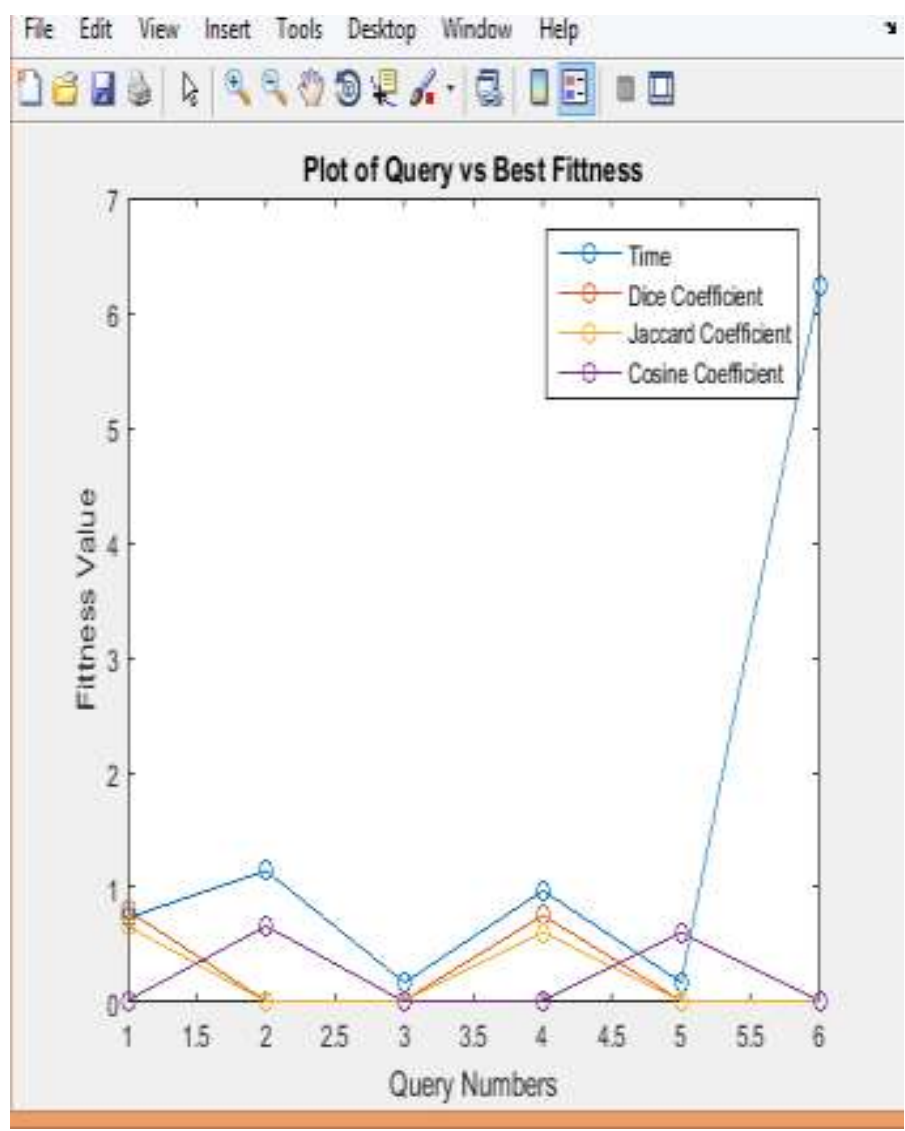

Figure $5 b$. Comparative segmentation results of the six algorithms on bladder Image 3. 
Table 1: The mean result for various algorithms applied on the images.

\begin{tabular}{|c|c|c|c|c|}
\hline Image & Method & Dice coefficient & $\begin{array}{c}\text { Jaccards } \\
\text { Coefficient }\end{array}$ & $\begin{array}{c}\text { Cosine } \\
\text { Coefficient }\end{array}$ \\
\hline \multirow{6}{*}{$\begin{array}{c}\text { Image } \\
1\end{array}$} & K-Means & 0.7972 & 0.6627 & 0.0031 \\
\hline & Fuzzy & 0.0078 & 0.0039 & 0.0009 \\
\hline & Prewitt & 0.0195 & 0.0157 & 0.0047 \\
\hline & Sobel & 0.7561 & 0.6078 & 0.0032 \\
\hline & Thresholding & 0.0117 & 0.0098 & 0.0009 \\
\hline & Fuzzy C-Means & 0.0195 & 0.0078 & 0.0012 \\
\hline \multirow{6}{*}{$\begin{array}{c}\text { Image } \\
2\end{array}$} & K-Means & 0.6966 & 0.5345 & 0.0012 \\
\hline & Fuzzy & 0.0086 & 0.0043 & 0.0007 \\
\hline & Prewitt & 0.0215 & 0.0172 & 0.0044 \\
\hline & Sobel & 0.6822 & 0.5176 & 0.0025 \\
\hline & Thresholding & 0.0129 & 0.0108 & 0.0009 \\
\hline & Fuzzy C-Means & 0.0215 & 0.0086 & 0.0007 \\
\hline \multirow{6}{*}{$\begin{array}{c}\text { Image } \\
3\end{array}$} & K-Means & 0.7972 & 0.6627 & 0.0024 \\
\hline & Fuzzy & 0.0078 & 0.0031 & 0.0012 \\
\hline & Prewitt & 0.0195 & 0.0157 & 0.0041 \\
\hline & Sobel & 0.7561 & 0.6078 & 0.0031 \\
\hline & Thresholding & 0.0117 & 0.0098 & 0.0014 \\
\hline & Fuzzy C-Means & 0.0195 & 0.0078 & 0.0013 \\
\hline
\end{tabular}

Table 2: Average Time Evaluation

\begin{tabular}{|c|c|c|c|c|c|c|}
\hline & $\begin{array}{c}\text { K-means } \\
(\mathrm{secs})\end{array}$ & $\begin{array}{c}\text { Fuzzy } \\
(\mathrm{secs})\end{array}$ & $\begin{array}{c}\text { Prewitt } \\
(\mathrm{secs})\end{array}$ & $\begin{array}{c}\text { Sobel } \\
(\mathrm{secs})\end{array}$ & $\begin{array}{c}\text { Thresholding } \\
(\mathrm{secs})\end{array}$ & $\begin{array}{c}\text { Fuzzy } \\
\text { C-Means } \\
(\mathrm{secs})\end{array}$ \\
\hline Image 1 & 1.2311 & 1.1062 & 0.19742 & 1.00175 & 0.19054 & 6.3868 \\
\hline Image 2 & 0.96111 & 1.0985 & 0.22655 & 1.0448 & 0.22834 & 3.8221 \\
\hline Image 3 & 0.77335 & 1.3184 & 0.17846 & 0.9248 & 0.16531 & 5.710 \\
\hline
\end{tabular}

\subsection{Discussion}

Comparison of different methods should not be done blindly as performance depends largely on metrics used as different metrics reflects different aspects of the algorithms' character. Therefore, the evaluation is done based on metric reflections.

As shown in Table 1, K-Means and Sobel showed a very high dice coefficient values in the experiment, with the k-means method giving the best performance. The Dice coefficient, D measures the extent of spatial overlap between two binary images. It is commonly used in reporting performance of segmentation and gives more weighting to instances where the two images agree. Its values range between 0 (no overlap) and 1 (perfect agreement) so the higher the dice co-efficient, the higher the algorithm's accuracy. 
The Jaccards co-efficient also measures similarities and provide the same metric as the Dice but in a similar manner and so, the higher the values the better the performance of the algorithm. And from Table 1, K-Means and Sobel still are on the high side respectively thus outperforming the others.

The cosine similarity is being used for the angular distance, and measures angular similarity coefficient in image patterns whose resulting function is a proper distance metric. With cosine coefficient, the Prewitt method achieved the best value, while the Sobel method ranked close followed by K-Means, thresholding, Fuzzy and Fuzzy C-Means.

But in terms of computing time as shown in Table 2, Prewitt algorithm performed faster than the rest while Fuzzy C-Means used the most of time.

\section{CONCLUSION}

The increasingly important role of medical imaging in the diagnosis and treatment of disease has opened an array of challenging problems. In this research, a set of tests was carried out on different bladder images to verify the accuracy of six commonly used image segmentation algorithms in three categories using a series of similarity metrics. The results indicate that the K-Means (a clustering technique) produced the most accurate result with respect to two of the similarity metrics used with Sobel (an edge detection method) following. The Prewitt method (also an edge detection technique) outperformed others when evaluated with the cosine coefficient while also having the fastest computational time. This means that the latter combines both accuracy strength and speed compared to the other evaluated algorithms.

However, it is worth mentioning that more factors other than accuracy should be considered in the evaluation of segmentation algorithms such as computational complexity, requirement of user intervention.

\section{ACKNOWLEDGEMENT}

We wish to acknowledge the efforts of Aduroja Adewale Gabriel and Adeniran Opeyemi Taiwo, B.Tech. Computer Engineering graduates of the Department of Computer science and Engineering, Ladoke Akintola university of Technology, Nigeria whose contributions to the research work is immense particularly in the experimental part of the work.

\section{REFERENCES}

[1] Takumi Uemura, Gou.Koutaki. Keiichi Uchimura. 2011. Image Segmentation Based on Edge Detection Using Binary Code 7(10). International Journal of Innovative Computing, Information and Control.

[2] M.S. Yang. 1993. "A Survey of Fuzzy Clustering, Mathl.ComputingModelling, vol.18, No.11, pp.1-16.

[3] Priya Sharda and Harbinger Singh. 2015. Performance Analysis of Medical Image Segmentation using Hybrid Technique. International Journal of Advanced Research in Computer Science and Software Engineering Volume 5, Issue 6 available at ww.ijarcsse.com.

[4] Salem Saleh Al-amri1, N.V. Kalyankar and Khamitkar S.D. 2010. Image Segmentation by Using Thershod Techniques. Journal of computing, volume 2, issue 5 pp: 83-86.

[5] Y. J. Zhang, "An Overview of Image and Video Segmentation in the last 40 years", Proceedings of the 6th International Symposium on Signal Processing and Its Applications, pp. 144-151, 2001.

[6] Ankit Chadha, Neha Satam. 2013. A Robust Rapid Approach to Image Segmentation with Optimal Thresholding and Watershed Transform. International Journal of Computer Applications. Volume 65- No.9, pp: 1-7.

[7] Senthilkumaran and R. Rajesh. 2009. Edge Detection Techniques for Image Segmentation - A Survey of Soft Computing Approaches International Journal of Recent Trends in Engineering, Vol. 1, No. 2, pp:250-254.

[8] Muthukrishnan.R and M. Radha. 2011. Edge Detection Techniques for Image Segmentation. International Journal of Computer Science \& Information Technology (IJCSIT) Vol 3, No 6:259-267.

[9] Raman Maini \& Himanshu Aggarwal. 2009. Study and Comparison of Various Image Edge Detection Techniques. International Journal of Image Processing (IJIP), Volume (3): Issue (1) 1 Pp: 1-12.

[10] Tikeshwar Gajpal and Sachin Meshram. 2013. Comparison of Traditional Edge Detection Techniques with Novel Fuzzy Approach in Noisy Medium. International Journal of Emerging Trends in Engineering and Development Issue 3, Vol.4, pp: 92-100.

[11] Jaskirat Kaur, Sunil Agrawal, Renu Vig. 2012. A Methodology for the Performance Analysis of Cluster Based Image Segmentation. International Journal of Engineering Research and Applications (IJERA), Vol. 2, Issue 2, pp.664-667.

[12] R. Manavalan \& B. Sathya. 2011. Image Segmentation by Clustering Methods: Performance Analysis. International Journal of Computer Applications. Volume 29 No.11, pp: 27-32. 